\title{
Continuing Medical Education
}

\section{Perioperative hemostasis and thrombosis}

Keyvan Karkouti MD MSc FRCPC, ${ }^{*} \dagger$ Kathleen M. Dattilo MD FRCPC $\dagger$

$\mathrm{P}$ ERIOPERATIVE hemostasis and thrombosis is an evolving topic of great relevance to anesthesiologists (and their patients) because patient outcomes are highly dependent on decisions that are made. Optimal perioperative management can reduce the risk of severe adverse events such as massive blood loss, arterial or venous thromboembolism, and death. Control of hemostasis and thrombosis requires attention throughout the entire perioperative period, and can best be accomplished using an interdisciplinary approach, with the involvement of surgeons and hematologists, and the anesthesiologist taking on the coordinating role as the specialist in Perioperative Medicine. To fulfill this role, anesthesiologists need to have, at a minimum, a working knowledge of normal hemostasis, as well as a thorough knowledge of the effects of major surgery and trauma on the hemostatic system. They need to know how to optimally manage patients with both normal and abnormal hemostasis during the perioperative period. Despite the importance of this rapidly evolving topic, anesthesiologists receive little formal training in this area. Consequently, this Continuing Education Module will focus on several important issues, hoping to encourage and facilitate an ongoing and comprehensive self-directed learning process in perioperative hemostasis and thrombosis. For the most part, the Module will focus on articles in the June, 2006 Canadian Journal of Anesthesia supplement entitled 'The management of bleeding and thrombosis in the perioperative period: the anesthesiologist's perspective.'

\section{Normal hemostasis - a fine balance}

Normal hemostasis involves a fine and complex balance between numerous anticoagulant and procoagulant components, the details of which are still not fully understood. Fortunately, anesthesiologists need not have a comprehensive understanding of the myriad pathways and components of the hemostatic system in order to effectively manage patients with normal or abnormal hemostasis during the perioperative period.

Drs. Lasne and associates present an excellent overview of the normal workings of the hemostatic system and some of the ways it can function abnormally. ${ }^{1}$ They highlight our current understanding of the three main components of the hemostatic system - primary hemostasis, plasma coagulation, and fibrinolysis - and the interactive role of the multiple cell types and proteins that take part in the process. For practical implications of these factors, we need to turn to other articles.

\section{Massive blood loss - underestimate its impor- tance at your (patients') peril}

Massive blood loss necessitating large volume fluid and blood product resuscitation is a common complication of major surgery and trauma, and constitutes an independent risk factor for serious adverse events including disseminated intravascular coagulation, organ dysfunction, and death. ${ }^{2,3}$ Resuscitation of massive blood loss - whether controlled (elective surgery) or uncontrolled (trauma or emergency surgery) - results in marked physiological perturbations. The primary objectives of resuscitating a massively bleeding patient are to rapidly restore or maintain normal circulating blood volume and to provide adequate tissue oxygen delivery. Although many questions remain unanswered, there is increasing evidence that the way these objectives are achieved (i.e., the resuscitation process itself) can influence morbidity and mortality. The article by Drs. Hardy and associates discusses some of these practical issues, including the pathophysiology of coagulopathy in a massively bleeding patient, and

From the Department of Anesthesia and Pain Management, * Toronto General Hospital, University Health Network, University of Toronto; and the Department of Health Policy, Management, and Evaluation, $†$ University of Toronto, Toronto, Ontario, Canada. Address correspondence to: Dr. Keyvan Karkouti, University Health Network, Toronto General Hospital, Department of Anesthesia, EN3-402, 200 Elizabeth Street, Toronto, Ontario M5G 2C4, Canada. Phone: 416-340-5164; Fax: 416-340-3698;

E-mail: Keyvan.karkouti@uhn.on.ca 
the importance of choice of fluid, hypothermia, and bemodilution on the extent of this coagulopathy. ${ }^{2}$

\section{Refractory blood loss - don't throw in the towel just yet}

The mainstay of medical management for patients with excessive blood loss has changed very little since the 1960s and is essentially limited to blood component therapy to replenish red blood cells, platelets, and coagulation proteins. Unfortunately, in a small but substantial proportion of coagulopathic bleeding surgical patients, blood component therapy fails to stop or reduce blood loss in a timely manner, and this almost invariably results in massive amounts of blood loss and very poor outcomes.

One of the more exciting recent developments in management of refractory blood loss has been the development of recombinant activated factor VII, a hemostatic agent originally developed as an alternate therapy for hemophiliac patients with inhibitors against factors VIII and IX. It is currently not approved for use as a rescue therapy for patients with refractory hemorrhage in non-hemophiliacs. Its safety and efficacy for this indication have not been assessed by randomized controlled clinical trials. Nevertheless, there is an increasing body of published experience that provides valuable guidance for use of this potent and potentially life-saving hemostatic agent as rescue therapy for non-hemophiliac patients with refractory hemorrhage. ${ }^{4,5}$

Managing patients at increased risk of thrombosis There are many surgery and patient related factors that increase the risk of postoperative arterial and venous thrombotic complications. To reduce this risk, the anesthesiologist must pay attention to the entire perioperative period and take into consideration patients' baseline risk, available prophylactic strategies, and their influence on anesthetic management.

Many patients who are at increased risk for arterial thrombosis present to the anesthesiologist on anticoagulant or anti-platelet drugs. The management of these patients is particularly challenging given that the risk of thrombosis must be balanced against that of perioperative hemorrhage. The article by Lecompte and Hardy provides a comprehensive review of antiplatelet drugs and makes practical suggestions on how to minimize the risks of both perioperative thrombosis and hemorrhage in patients who are on anti-platelet drugs. ${ }^{6}$

Multiple therapies are available for venous thromboembolism prophylaxis to patients at high-risk. Some of these therapies present a challenge to the anesthesiolo- gist and can influence anesthetic management. The article by Motte and associates provides a detailed yet practical overview of available prophylactic regimens, and guidelines based on the patients' risk for venous thromboembolism. ${ }^{7}$

Finally, an important prothrombotic condition, and one that is highly relevant to the anesthesiologist, is heparin-induced thrombocytopenia. It occurs frequently, is difficult to manage during the perioperative period, and can lead to grave complications. Dr. de Maistre and associates provide a thorough and timely review of this evolving topic. ${ }^{8}$

\section{Concluding remarks}

One important caveat to consider when reading the recommended review articles is that, by necessity, they are based on limited, often low-grade, evidence. As perioperative hemostasis and thrombosis is now a topic of great research interest, however, one can expect rapid and frequent advances in our understanding, and clinical management, of conditions that impair the hemostatic system during the perioperative period. Hence, ongoing self-directed learning is critical for clinicians who wish to stay abreast of these advances in this rapidly evolving and highly clinically relevant area.

\section{Specific objectives of the CME module}

The selected review articles should allow the reader to attain the following objectives:

1. Understand the mechanisms of normal and abnormal hemostasis

2. Understand the importance of massive blood loss as a perioperative complication

3 . Appropriately manage patients with massive perioperative blood loss

4. Identify and manage patients at high-risk for perioperative arterial or venous thrombotic complications

5. Recognize the limitations of existing evidence on perioperative hemostasis and thrombosis

\section{References}

1 Lasne D, Jude B, Susen S. From normal to pathological hemostasis. Can J Anesth 2006; 53(6 Suppl): S2-11.

2 Hardy JF, De Moerloose P, Samama M; Groupe d'Intérêt en Hémostase Périopératoire. Massive transfusion and coagulopathy: pathophysiology and implications for clinical management. Can J Anesth 2004; 51: 293310.

3 Holte K, Sharrock NE, Keblet H. Pathophysiology and clinical implications of perioperative fluid excess. $\mathrm{Br} \mathrm{J}$ Anaesth 2002; 89: 622-32. 
4 Karkouti K, Yau TM, Riazi S, et al. Determinants of complications with recombinant factor VIIa for refractory blood loss in cardiac surgery. Can J Anesth 2006; 53: 802-9.

5 Ozier $\Upsilon$, Schlumberger S. Pharmacological approaches to reducing blood loss and transfusions in the surgical patient. Can J Anesth 2006; 53(6 Suppl): S21-9.

6 Lecompte T, Hardy JF. Antiplatelet agents and perioperative bleeding. Can J Anesth 2006; 53(6 Suppl): S103-12.

7 Motte S, Samama CM, Guay J, Barre J, Borg JY, Rosencher N. Prevention of postoperative venous thromboembolism. Risk assessment and methods of prophylaxis. Can J Anesth 2006; 53(Suppl): S68-79.

8 de Maistre E, Gruel $\Upsilon$, Lasne D. Diagnosis and management of heparin-induced thrombocytopenia. Can J Anesth 2006; 53(6 Suppl): S123-34. 